REVIEW

\title{
Nutriproteomics: Facts, concepts, and perspectives
}

\author{
Sascha Sauer and Toni Luge \\ Otto Warburg Laboratory, Max Planck Institute for Molecular Genetics, Berlin, Germany
}

\begin{abstract}
Nutrition is a basic component of life. Nowadays, human nutrition research focuses amongst others on health-related aspects of food ingredients and extracts, and on analyzing the outcomes of specific diets. Usually, food ingredients such as bioactive peptides come in complex matrices. Single compounds, multiple interactions thereof and the underlying food matrix can vary physiological response of the organism. Proteins and peptides derived from food and beverages can cause adverse allergic reactions but are in general required for multiple functions such as growth and homeostatic regulation. Endogenously expressed human proteins and peptides can be used as biomarkers to monitor physiological deregulation and the effects of food consumption. The intestinal microbiome seems to play a fundamental role in establishing and maintaining physiological regulation and in digesting proteins and peptides and other biomolecules derived from food. Notably, the subtle interplay of flavor naturals in food and beverages with olfactory receptors can result in establishing human taste preferences, which again influences overall physiology. This article presents basic approaches and concepts to address scientific questions in nutritional proteomics and discusses potential benefits of proteomics-based methodologies to help advance the field of molecular nutrition research.
\end{abstract}

\section{Keywords:}

Diagnostics / Food science / Mass spectrometry / Proteogenomics / Systems biology

\section{Introduction}

Nutrition has a major impact on human health. On the one hand side, large proportions of the world population still suffer from malnutrition and lack of major nutrients. In contrast, sedentary populations that consume high-calorie diets are affected by so-called western life-style associated disorders such as obesity and type 2 diabetes [1].

Current nutrition research aims to gain insights in the physiological role of minor dietary constituents such as secondary metabolites and bioactive peptides. An additional focus lies on the development of methods to monitor dietary interventions by using biomarkers (Fig. 1) [2,3]. Further important current topics that are discussed in this article comprise the interaction of the human intestinal microbiome and food, and the development and establishment of taste preferences (Fig. 1).

Over the last decades hypothesis-driven nutrition research revealed causative molecular mechanisms of individual

Correspondence: Dr. Sascha Sauer, Otto Warburg Laboratory, Max Planck Institute for Molecular Genetics Ihnestrasse 63-73, 14195 Berlin, Germany

E-mail: sauer@molgen.mpg.de

Fax: $+49-30-84131960$ components of food in controlled experimental set-ups. However, in many cases in vitro and in vivo experiments did not fully reflect the complexity of food matrices. Moreover, active biomolecules derived from food usually do not produce strong effects as known from optimized pharmaceutical compounds. Furthermore, it is difficult to extrapolate results from in vitro or model animal based studies to humans. On the other hand, epidemiological studies in humans that analyzed the effects of diets or food ingredients usually generate correlations but rarely causative explanations [4]. All these mentioned problems resulted in part in fragmented pieces of incomprehensive knowledge.

Modern nutrition research promises to translate empirical knowledge to evidence-based science [5]. However, due to the inherent complexity of food and human physiology, this is difficult to achieve. Nutritional proteomics or nutriproteomics comprises a synthesis of comprehensive information on proteins from three different areas: host organism, food, and resident microorganisms [6]. The proteome of the host and the metaproteome of its intestinal and other microorganisms that are located elsewhere in the body (e.g. in the mouth) permanently react in response to changing environmental conditions such as incoming food. In this context, cellular

Colour Online: See the article online to view Figs. 2 and 3 in colour. 


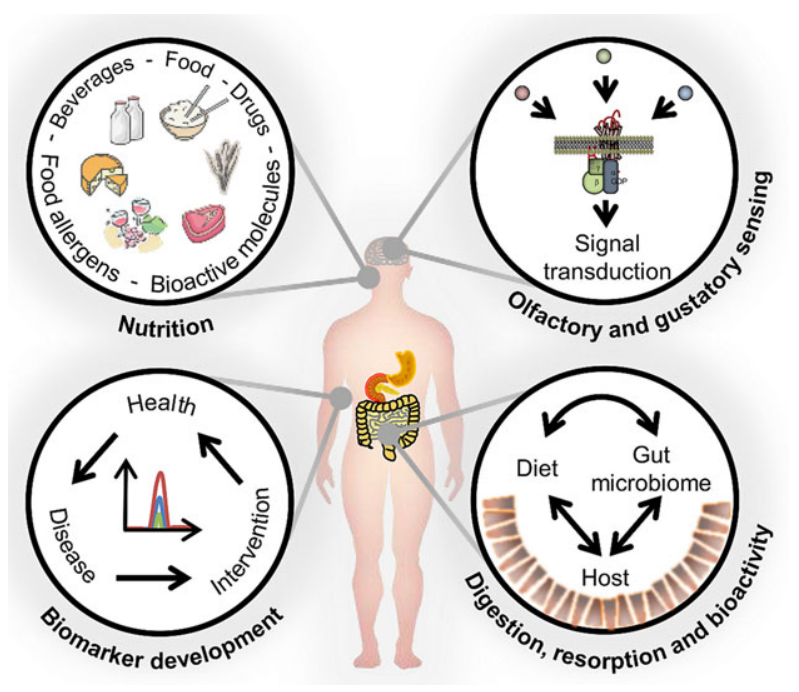

Figure 1. Proteomics can contribute to important areas of modern nutrition research. In general, nutrition can exert various complex physiological effects, which is being analyzed by many scientific approaches and methodologies including proteomics. In this article, we focus on four main areas of research. One major focus of current nutriproteomics research concerns the detection of bioactive peptides. Secondly, development of biomarkers is an important topic to eventually provide evidence for efficient nutrition-based disease prevention or treatment programs. Furthermore, we present metaproteomics approaches to study the interference of diets with the gut microbiome. Eventually, as a paradigm of the complexity of nutrition research, we focus on olfactory and gustatory sensing of food ingredients, and outline potential future lines of research in physiology.

processes entailing deregulation in protein abundance and posttranslational modifications are highly dynamic [7].

The fruitful application of proteomics to gain insights in addition to conventional or other "omics" approaches is a current concern. Here, we mainly aim to provide a brief overview on "success stories" of first nutriproteomic applications, which shed light on the role of dietary proteins and peptides. Secondly, we discuss biomarker strategies to detect physiological variation derived from nutrition. We further propose conceptual aspects of applying proteomics to adequately analyze complex nutritional effects, including the interaction of the human microbiome and nutrition. Finally, by presenting a major field of nutrition research - the development of taste preferences - we sketch general ideas to analytically grasp multilayered biological systems.

\section{Food-derived peptides and proteins}

Proteins are the only nitrogen and essential amino acid source for the human organism. In general, proteins provide key building blocks for protein homeostasis and their intake contributes to protein turnover balances in the body. Nutritional proteins and peptides can generate adverse and beneficial effects. In general, food proteins differ from human proteins in the composition of amino acids. Animal sources such as egg or meat have an amino acid pattern that is closer to the requirements of human physiology than plant-based sources. But in general also a well-balanced vegetarian diet will deliver sufficient amounts of required amino acids. For any nutritional protein or peptide in order to exert physiological activity, it must be digestible and reach a target site in the body at a sufficient concentration. In this context, it is important to understand factors that influence the release of active peptides from the food matrix and their fate in the organism.

Adverse, synergistic or competitive effects of food ingredients, as well as their potentially weak long-term effects, are much less controllable compared to drug treatments. These challenges make it in part difficult to develop evidence-based targeted molecular nutritional intervention [8].

\subsection{Allergy causing peptides and proteins}

Food allergy or milder forms such as food intolerance are adverse reactions to natural or processed food or food additives. Nowadays, about 250 million people worldwide suffer from food-based hypersensitization [9]. Typical symptoms of food allergies comprise urticarial, vomiting, asthma and anaphylaxis. Food allergies are in general caused by proteins. Allergic reactions are routinely being analyzed by determining the physiological levels of IL-10, TGF $\beta$, PD-1, CTLA-4, and IgE [6]. Discovery of nutritional ingredients that induce allergic reactions seems to have a significantly growing impact on human health [10].

Eight groups of food (termed "the Big 8") that cause allergic reactions have been defined: egg, fish, milk, peanut, shellfish, soy, tree nuts, and wheat. Altogether approximately 600 food allergens have been found of which 206 are officially registered by the International Union of Immunological Societies (http://www.allergen.org).

Detecting and quantifying known allergens derived from food remains difficult in routine due to different food sources and complex food matrices, as well as often observed multiple allergens in a food sample [11]. Food labeling is regulated by law but due to a lack of sufficiently standardized procedures this requirement cannot be properly applied in daily practice. Current procedures for detecting allergens in food are based on enzyme-linked immunosorbent assay (ELISA) or lateral flow devices [11]. Methods like ELISA are usually restricted to single known allergens and require target protein specific antibodies, which can cross-react and might interfere with various compounds in food such as large secondary metabolites (polyphenols, tannins). Moreover, food processing can denature allergic proteins potentially resulting in false-negative results of antibody-based analysis. In general, ELISA-based kits suffer from poor standardization and reproducibility of results between different kits [12].

Emerging mass spectrometry methods enable easy sample preparation and rapid analysis of multiple known allergens at a time. These robust and sensitive methods might fulfill more adequately the strict requirements for proper 
legislation of law than conventional antibody-based methods. Presently, unique signature peptides from about 40 different allergens have been identified by mass spectrometry [11]. Moreover, multiple reaction monitoring (MRM), a peptidefragmentation based analysis method, allows for absolute quantification of (signature) peptides derived from allergenic proteins. MRM can detect peptides even down to the attomole range, similar to ELISA [13]. For reliable quantification three peptides should be selected per allergenic protein, including isotopically labeled peptides as standards to enable absolute quantification. This set-up step requires optimization for peptide fragmentation but is usually doable in a reasonable time.

Initial discovery of unknown allergens in food and characterization has typically been performed by identification of IgE-binding proteins using inexpensive and fast immunoblot-coupled techniques based on two-dimensional polyacrylamide gel electrophoresis (2D-PAGE) [14]. Recent methods based on immunoaffinity capillary electrophoresis (IACE) coupled with matrix-assisted laser desorption/ionization mass spectrometry (MALDI MS) required only few microliters of blood to detect IgE. Thereby, the effects of potential food allergens from milk could be efficiently analyzed in a so-called component-resolved diagnostic approach [15].

In more conventional targeted mass spectrometry approaches grain-specific peptide markers for the detection of gluten (and allergic gliadin peptides thereof) in flour were recently discovered and validated [16]. Furthermore, a number of new protein-derived food allergens, many of them glycoproteins in the analytically amenable size-range of 14 to $40 \mathrm{kDa}[17,18]$, have been identified in fish, eggs, milk, wheat, peanuts, tree nuts, citrus fruits, and sesame seeds. A number of additionally potentially allergic substances derived from food are currently under study, and may be added to abovementioned diagnostic panels after extensive validation.

However, although many allergenic structures derived from food and their effects are known, the mechanisms underlying allergic reactions still remain poorly understood, making medical treatments difficult. Recommendations are mainly restricted to avoid consumption of specific ingredients. Suitable models for studying the role of proteins and their glycosylation in food allergy are needed to better understand the allergenic role of proteins [19].

\subsection{Bioactive nutritional proteins and peptides from milk}

Bioactive peptides have been described as "specific protein fragments that have a positive impact on body functions or conditions and may ultimately influence health" [20]. Bioactive peptides have been studied over the years, leading to large data repositories and tools that can be used for classification and for analyzing potential bioactivities [21,22].

Bovine and human milk are known as well-analyzed rich resources of bioactive molecules. A number of bioactive peptides are released by proteases in milk, by microbial fermentation, in the gastrointestinal tract, or a combination of mentioned processes [23]. Interestingly, these peptides can influence a number of physiological events. Notably, in particular bovine, sheep, and goat milk represents nutrition that has coevolved with mankind.

The protein complement of human milk consists mainly (50:50) of casein and whey. In contrast, bovine milk contains $80 \%$ caseins and $20 \%$ whey [23]. Caseins are ion carriers and precursors of bioactive peptides, whereas whey proteins support immune modulation and defense [24]. Human milk is the gold standard for neonate and infant nutrition. Beside caseins major constituents are $\alpha$-lactalbumin, lactoferrin, albumin, and immunoglobulins, which altogether account for more than $99 \%$ of the entire protein content. The residual $1 \%$ fraction comprises a mixture of bioactive proteins and peptides, which are still largely unexplored. D'Auria et al. have systematically explored the proteome set-up of several mammalian species (human, bovine, goat, horse, donkey, and water buffalo milk) with the intention to optimize infant milk substitutes [25]. Beside protein composition, a number of studies sought to identify bioactive proteins and peptides in milk using more or less standard fractionation and proteomics techniques. Many of these studies focused on the detection of bioactive milk peptides by in vitro digestion processes using bacterial cultures [26-28] or isolated proteases [29]. These studies led to the identification of proteins and bioactive peptides with various functions in dairy products, as well as to the establishment of quality assessment parameters for dairy-based products.

Furthermore, promotion of host immune defense mechanisms versus microbial infections due to the iron-binding glycoprotein lactoferrin have been described [30]. Soluble CD14 in milk seems to be involved in modulating innate and adaptive immune response including various modulating proteins in the neonatal intestine [31]. Interestingly, the proteolytic system of bacteria is used to generate milk fermentation products such as yoghurt or cheese, which can support liberation of peptides from a food matrix or processing of bioactive peptides from precursors [32]. This observation provides evidence for the potential of (traditional) food technology approaches to contribute to human nutrition. In addition to immune modulatory functions, a number of additional roles could be assigned to casein-derived peptides in a recent study on raw milk, including antimicrobial and antiamnestic activities, angiotensin converting enzyme inhibitory (antihypertensive) functions, interaction with calmodulin, antioxidant effects, and bitter taste [33]. These results are in line with a number of previous reports [34-40]. Moreover, opioid peptides were found to be encrypted in proteins such as milk casein. Once released such peptides can inhibit cysteine uptake and modulate antioxidant cellular capacity and results in epigenetic changes in a cell culture model [41]. High doses of casein intake may thus potentially influence early postnatal development. 
All the various studies in the field provided a very detailed understanding of the composition of proteins and peptides in milk and potential physiological effects of milk consumption. The insights gained from these studies can be exploited for tailoring diary product development. Further studies on the so far less studied low-abundant proteins and peptides in milk and in particular in other protein-rich nutritional sources may produce additional valuable basic knowledge.

However, the various above-mentioned in vitro digestion approaches cannot reveal the endogenously produced peptides that are present in milk. Only few attempts have been made to detect naturally occurring peptides, which derived from a rather low number of (abundant) proteins [42,43]. Recently, Guerrero et al. mechanistically analyzed the formation of bioactive peptides in human milk [44]. These authors made use of an efficient procedure to purify and analyze endogenous peptides in milk [45]. For peptide identification, the authors searched a human milk protein library composed of 975 entries allowing nonspecific protease cleavage. Altogether, Guerrero et al. found nearly 700 naturally occurring peptides derived from 30 human milk proteins. The majority of peptides derived from highly abundant $\beta$-casein and $\alpha_{\mathrm{s} 1}$-casein, and from two low abundant proteins, namely osteopontin and polymeric immunoglobulin receptor. Using computational tools such as Peptide Extractor (PepEx) [44], the authors analyzed site-specificity of proteolysis. By calculating local degrees of protein disorder with Disprot VL3E [46] they observed specific endogenous protease activity in the mammary gland and unexpected properties of substrates. Eventually, Guerrero et al. discovered striking intrinsic disorders of substrate proteins. Notably, these characteristics of substrate proteins can predefine proteolytic sites [44].

\subsection{Bioactive nutritional proteins and peptides from cereals and legumes}

A current emphasis in nutriproteomics lays on understanding the roles of plant-based proteins and peptides derived for example from protein-rich soybean, wheat and peas. Whereas nowadays in developed countries animal protein sources make up about $70 \%$ of diet, this was completely different in former times. Edible plants represent valuable alternatives for protein intake, which are much cheaper than animalbased nutritional proteins. Plant-based protein consumption leads in general to higher sustainability as less ecological resources have to be exploited. Still minor but nevertheless significantly changing consumer preference (vegetarian or vegan people) contributes to a trend of consuming plant-based protein sources. Main substitutes for meat and fish proteins include soy, legumes, and wheat, which have all been thoroughly characterized in terms of nutritional value, bioactive peptides, and other bioactive compounds or contaminants [6].

Wheat is one of the most important food resources. Breadmaking and nutritional qualities of wheat depend on protein composition of wheat seeds. A 2-D gel study identified 230 wheat proteins derived from different cultivars of which 36 were differentially expressed between two wheat varieties [47]. Soybean is a popular source of food proteins, which has traditionally been used in Asia. Soybean offers a complete spectrum of (essential) amino acids. A lot of health claims were made for soy, which were at least in part supported by proteomic analyses [48]. As other legume seeds, soybeans contain a large fraction of storage proteins, rendering detection of less abundant bioactive proteins or peptides difficult, which can in part be mediated by precipitation of storage proteins [49]. Biologically active proteins or peptides in soy comprise lunasin, Bowman-Birk inhibitor, lectin, and $\beta$-conglycinin [50]. Various beneficial effects were attributed to these bioactive molecules; for example, lunasin was found to inhibit core histone acetylation and cancer cell division [51]. Further information can be gained from established databases on the soybean proteome, which are mainly based on 2-D gel analyses [52]. Soybean products are also popular because of reported health benefits of other ingredients such as secondary metabolites including isoflavones and saponins [53,54]. In general, it seems that most of the health-beneficial effects that have been associated with soy-based diets could be predominantly attributed to phytochemicals rather than proteins or peptides in soy products. Nevertheless, for example Beermann et al. observed antioxidative properties of enzymatically released peptides from soy protein isolates [55]. Such peptides may offer opportunities to develop nutraceuticals to prevent and treat oxidative stress-related diseases or aging processes in general.

Interestingly, proteomics could trace potential environmental contaminations of plant material with healthbeneficial peptides. For example, Danchenko et al. analyzed soybeans by 2-D gels and mass spectrometry that were sown in the radioactively contaminated Chernobyl area and in noncontaminated control fields [56]. These authors reported 9.2\% of 698 quantified proteins to be differentially expressed. This approach may provide a basis for improving control of food and the quality of plant-peptide based nutraceuticals.

A number of further protein sources such as legumes (e.g. peas) [57] have been analyzed for bioactive peptides, most of them using mass spectrometry technologies based on 2-D gels. It seems that by using more recent nano-LC based methods further, in part complementary details on the role of bioactive proteins and peptides from these sources can be uncovered to improve our basic knowledge. Such increasingly growing background information on the composition of bioactive peptides in food may eventually result in the design of efficient strategies to tailor nutritional interventions.

\subsection{Technical issues in bioactive peptide and protein discovery strategies}

Detection of peptides derived from proteins of food requires adaptation of common analytical workflows. In general, the peptidome (all peptides in a given biological sample) 


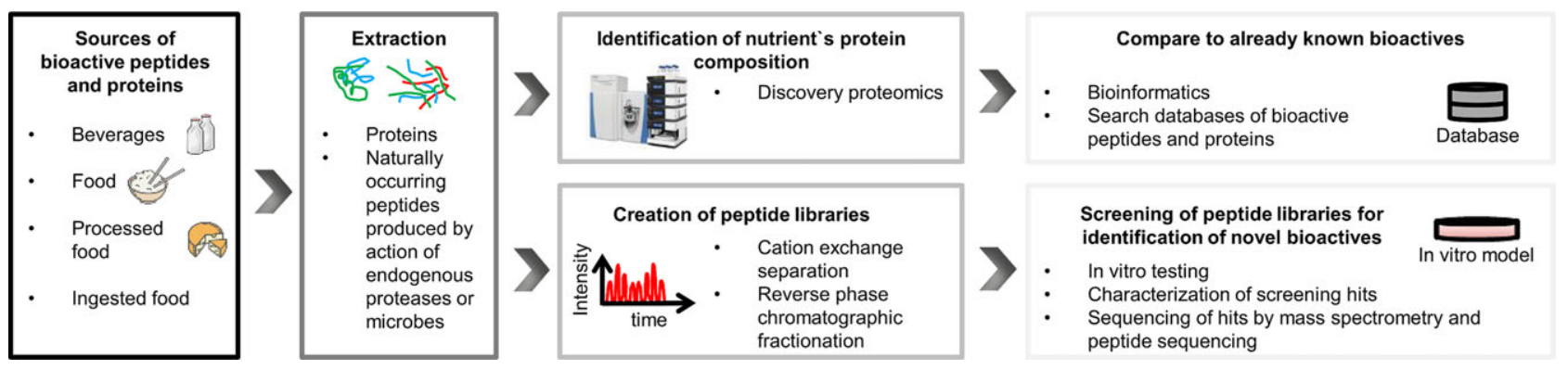

Figure 2. Discovery of bioactive peptides and proteins from nutritional sources. Beverages, food, and processed food (like cheese) are interesting resources of peptides that may exert diverse effects on the human body after ingestion. In conventional approaches the protein and peptide composition of nutrients is determined by discovery proteomics. Potential bioactive protein-based ingredients can be revealed by comparisons to already established peptides and proteins with bioactive functions. Another promising strategy aims to identify novel physiologically relevant bioactive peptides [62]. Nutrition such as milk is available in vast amounts, which enables the creation of peptide libraries by isolating the protein complement and massive cation exchange and reverse phase chromatographic fractionation. The obtained libraries can be screened in test systems of interest, and positive hits further characterized with proteomic technologies. For instance, LC$\mathrm{MS} / \mathrm{MS}$ or Edman degradation can be used to determine the sequence of the peptides, which is prerequisite for peptide synthesis and further validations.

comprises fragments of peptides derived from proteolysis of proteins. The peptidome and the proteome are linked by the activity of proteases (exopeptidases and endopeptidases), of which in the human genome more than 500 are encoded. The action of proteases can be further modulated by protease activators and inhibitors.

Most proteolytic peptides vary in their chemical properties from the tryptic peptides generated in typical proteomics experiments. Due to their variable mode of action endogenous proteases can produce a large variety of peptide lengths, sequences, terminal residues, and chemical modifications of peptides [58]. To facilitate the identification of unspecifically digested proteins novel, robust and reliable performing algorithms for bioinformatic analysis of fragment spectra need to be developed. Promising approaches include de novo sequencing $[59,60]$ and protease unspecific database searches [61]. De novo peptide sequencing from fragment ion spectra omits the need for creating and matching fragment ion lists generated from protein sequence databases by predefined rules, such as tryptic cleavage, thus enabling the identification of atypical digested peptides. In protease unspecific database search approaches, fragment ion lists for all possible cleavage sites are deduced from protein sequence databases. In this way both of the peptide termini can be arbitrary positions in a protein, which leads to a much larger search space than by applying only one specific digestion rule, thereby increasing the possibility of false identifications. In the aforementioned study on endogenous milk peptides, Guerrero et al. [44] applied a rather small database with 975 milk protein entries instead of a large human reference proteome database to counteract this issue. However, to date both approaches require high quality data to achieve reliable results. To reduce errors manual inspection of spectra is thus still often necessary. Further technology development will be required to provide efficient tools for analyzing bioactive peptides of the complex food peptidome.
Interestingly, peptide libraries of proteolytically degraded proteins or polypeptides can nowadays be derived from human or animal sources such as body fluids or tissues [62]. These resources rely on ultracentrifugation and cation exchange followed by reverse-phase chromatography fractionation of complex peptide mixtures (Fig. 2). Such peptide libraries were applied in cell culture-based screenings for the identification of unknown bioactive peptides in human plasma, milk, placenta, sweat, seminal plasma, and saliva [62]. Clearly, these new resources can provide useful means to also discover bioactive peptides derived from external sources such as food.

\section{Protein and peptide based biomarkers}

Well-validated biomarkers are important for advancing in clinical diagnostics $[63,64]$. In general, a biomarker is defined as an observable molecule of an organism that indicates a physiological state. Biomarkers could deepen our understanding of the effects of diet [65] and provide informative links between diet and health or disease [58]. Biomarkers can eventually be used for diagnostics and monitoring of nutritional interventions. Biomarkers should generally be specific and easily applicable. In general, biomarkers can be gained from a variety of noninvasive samples derived from saliva, urine, feces, hair, nails, breast milk or expired air; or by invasive sampling of serum, plasma, red and white blood cells or peripheral blood mononuclear cells, bone marrow, and biopsies of tissues such as adipose tissue (Fig. 3). Clearly, to efficiently detect proteins or peptides, all these samples involve different sample preparation procedures and analytical challenges [66]. Early markers may offer greatest potential for nutritional intervention, whereas late markers are generally closer to the disease phenotype. As known from conventional clinical proteomics workflows, nutritional protein- or 


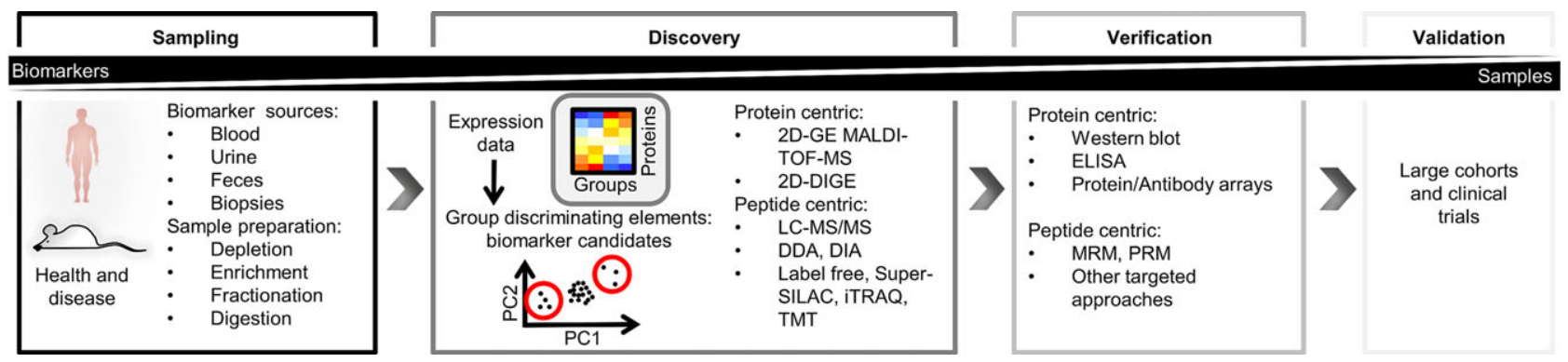

Figure 3. Biomarker development in nutrition science. Four main categories for establishing protein or peptide based biomarkers can be defined. First, easily accessible body samples have to be collected in a highly standardized manner. For example, samples such as serum need to be processed under well-controlled conditions to ensure comparability and reproducibility [150,151]. Sample processing such as depleting highly abundant, noninformative proteins or enrichment of low abundant proteins of potential interest can increase detection sensitivity. In the discovery phase, single markers or combinations of multiple biomarker candidates are identified to robustly predict group (e.g. health or disease) associations. For that purpose either protein centric methods, such as 2D-gel electrophoresis combined with MALDI-TOF-MS, or peptide centric mass spectrometry methods, such as shotgun proteomics by LC-MS/MS with either data dependent (DDA) or independent (DIA) acquisition methods, and a wide range of quantification technologies including for instance label free or ITRAQ (isobaric Tags for Relative and Absolute Quantitation), are applied. Next, biomarker candidates need to be verified in larger cohorts. Therefore, antibody based methods (western blot, ELISA, protein/antibody arrays) or targeted mass spectrometry based technologies, such as multiple reaction monitoring (MRM) or parallel (PRM) reaction monitoring, can be applied. Finally, rapid analysis kits can be developed to monitor and validate biomarker panels in clinical screenings of large, appropriately designed patient cohorts. For more details, the reader is referred to previous literature $[66,152-155]$.

peptide-based biomarker development usually includes discovery in few samples and verification phases in several hundred samples. Validation is subsequently performed in large, well-controlled cohort studies (Fig. 3) [67]. However, efficient application of biomarkers in a nutritional context still suffers from a lack of validation due to low observable effects such as small fold changes at an early disease state [68].

The use of potential biomarkers can be influenced by various factors that are difficult to control [69]. Studies on potential biomarkers that react to nutritional effects are compromised due to a rather poor understanding of bioavailability and mode of action of food ingredients. For example, timing of measurement after food intake is important if the marker is a result of nutrient bioavailability and efficacy. Absorption, distribution, metabolism and excretion of incoming nutrients may influence response of biomarkers. Also, homeostatic buffering to nutritional changes can result in poor response of biomarkers. Environmental factors and (epi-) genetic predisposition might play confounding roles in this context. Moreover, many food ingredients have multiple targets and only weak singular effects, making it difficult to establish molecular markers to predict outcomes of specific diets.

\subsection{Health and disease biomarkers}

To identify and validate potential health and disease biomarkers in a nutriproteomics context, first studies applied 2-D gels and mass spectrometry readout, and protein or antibody microarrays [70]. Later on, nano-liquid chromatography mass spectrometry methods for discovery-based and/or targeted approaches became more dominant.

Many initial nutriproteomic analyses in the years 2000 targeted various disorders like immunity, inflammation, allergy, and obesity/diabetes. These analyses were mainly performed in cell culture and/or model animals to determine effects of food ingredients [6]. For example, 30 differentially expressed proteins were observed in the colonic mucosa of mice fed with increased amounts of vegetables [71]. Only 6 of these proteins could eventually be identified by MALDI-MS. Nevertheless, the alteration of these few proteins could be aligned with their previously reported protective role versus colorectal cancer, showing potential colon cancer preventive effects by increasing amounts of vegetables in food. Furthermore, even earlier proteomic analyses including 2D gels of colon cancer cells grown in the presence or absence of the flavonoid flavone, an ingredient that is present in many fruits and vegetables [72], indicated amongst others induction of apoptosis in colon cancer cells. Furthermore, mass spectrometry analysis revealed the implication of diallyl disulfide, a prominent compound in processed garlic, in protein phosphorylation of the kinase ERK. Diallyl disulfide led to cell cycle arrest [73] and modification of thiol groups of the cytoplasmic protein Keap1 that senses - in concert with the oxidative stress response transcription factor Nrf2 - the redox state of the cell [74]. A number of other studies have applied similar approaches to characterize biomarkers which have been recently reviewed (for details the reader is for example referred to the comprehensive references 5 and 7).

\subsection{Advantages of comprehensive integrated analyses of endogenously expressed proteins}

In general, protein-based detection is closest to biological function compared to other biomolecules, including information on the localization of active molecules such as 


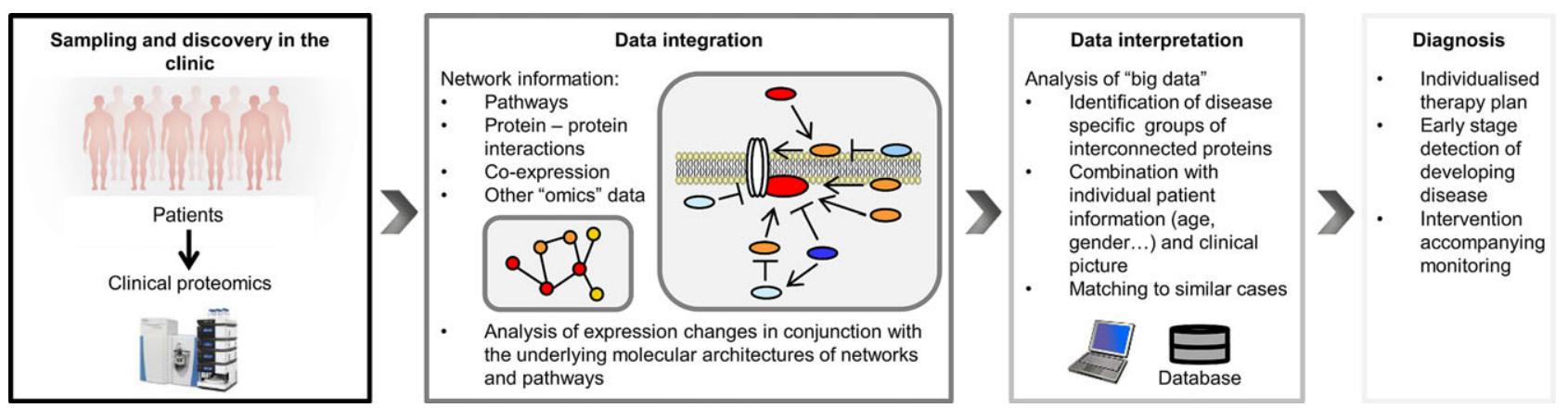

Figure 4. Emerging proteomic strategies in the clinic. Complex diseases can in many cases not be broken down to single causative and predictive elements. Therefore, clinical applications of proteomic technologies such as advanced, high-speed and robust sampling as well as LC-MS/MS approaches, hold great promise to infer underlying altered molecular architectures on a proteome-wide scale instead of relying on few single biomarkers. Furthermore, integrating protein expression data with additional medical information may enable powerful diagnosis of subtle physiological perturbations. Further progress on bioinformatics resources will enable clinicians to interpret large-scale, "big" data on an individual basis to guide patient monitoring. This development shall eventually allow for establishing individualized therapy to efficiently prevent and treat complex diseases.

secreted peptides (secretome). For example, recently the effects of calorie restriction on the secretome of human adipocytes was analyzed [75]. Interestingly, these data hinted to beneficial, reduced inflammatory processes in such cells. Moreover, using a transwell cell culture system, Freiwald et al. quantitatively analyzed the proteomes of proximal macrophages and adipocytes [76]. Thereby, subtle fine-tuning of transcriptome and protein pathways - but not of single key proteins - could be observed, without artificially boosting effects by using high doses of inflammatory triggers such as lipopolysaccharide. Adipocytes and macrophages showed a downregulation of energy-producing pathways and upregulation of cellular stress, cancer, and inflammation pathways. These observed effects could contribute to understanding of low-grade inflammation processes underlying diet-induced insulin resistance.

Furthermore, Meierhofer et al. detected several thousand proteins in various tissue samples such as adipose, liver, and heart tissue to analyze the effects of a high-fat diet on the proteome [77]. Notably, in this in vivo context most proteins were only slightly changed in expression, and the expression of individual genes and proteins showed only weak correlations with each other. However, when looking at pathways or sets of proteins, the correlations of gene and protein sets became more significant, suggesting that functional response to diet was rather a result of concerted effects of ensembles of biomolecules than a result of the action of single key regulators. Interestingly, in mice fed a high-fat diet and treated with the diabetes drug rosiglitazone, protein set analysis indicated down-regulation of oxidative phosphorylation in heart. Many of the proteins and enzyme activities of the oxidative phosphorylation chain were not significantly regulated (as shown by western blotting and enzymatic analyses). But the detected levels of metabolites as ATP in heart tissue were indeed lowered, indicating physiological deregulation of oxidative phosphorylation. Instead of relying on single or few markers, these data may argue for increased information content of ensembles of proteins, which can be used as profiles for robust diagnostic applications [78, 79].

In principle, proteomic approaches can be efficiently applied in nutrition research to contribute insights to physiological effects of nutrition and to discover and validate (sets of) biomarkers that can be used for accompanying nutritional interventions. Using information on the annotated pathway or the network level of proteins instead of focusing on only few markers is potentially a fruitful approach to adequately address the effects derived from complex food matrices or dietary interventions (Fig. 4). The information derived from protein sets may be complemented by integrating transcriptomics and metabolomics data [80]. Notably, protein networks that are regulated by effects of nutrition can lead to modification of chromatin. Such histone protein and DNA modifications can lead to differential expression of genes and eventually proteins [81]. Integrated "omics" analyses may allow us to better understand the plasticity of physiology to react to environmental changes based on nutrition (Fig. 4). As the underlying technologies for data generation and bioinformatics tools are rapidly evolving, such data integration may become useful for routine diagnostics in the mid-term future. Main disease areas under study currently comprise metabolic diseases (obesity, insulin resistance, type 2 diabetes), chronic inflammation (including inflammatory barrier diseases), and allergy.

In summary, proteomics and other "omics" data including also genetic and other patient-specific information can support the establishment of assembled individualized data to tailor recommendations to rationally optimize physiological effects by stratifying nutritional intervention.

\subsection{General methodological problems in biomarker-based nutriproteomics research}

In nutriproteomics, hypothesis-free discovery proteomics and hypothesis-driven targeted approaches are being applied as 
in other fields of the life sciences [7]. In principle, more or less the same standard - mostly mass spectrometry based proteomics approaches can be useful [82]. Clearly, also nutrition science would benefit from general methodological advances. This comprises detecting the full sequence of proteins in a sample including all posttranslational modifications and quantitative analysis over the entire biological dynamic range (probably 12 orders of magnitude and not the current 2-7 orders of magnitude that can be detected depending on the proteomics method applied). A further goal consists in boosting sensitivity down to the level of single molecules $[83,84]$.

Proteomics based analyses of posttranslational modifications still only hit the tip of the iceberg [85]; for example, although revealing new phosphosites in many studies, mass spectrometry based methods often do not detect the majority of phosphorylation sites involved in cellular protein signaling cascades and miss key-regulatory sites. Moreover, analysis of posttranslational modification data is also lacking powerful tools for specifically extracting potentially causative factors [86]. Recent bioinformatics tools such as PHOXTRACK might provide new avenues to alleviate this current problem to efficiently interpret large data sets of posttranslational modifications (see: http://phoxtrack.molgen.mpg.de) [87].

In principle, detection of individual cells - and not a bulk of cells - would allow analyzing underlying distributions of protein expression and modification [88]. As in single cell transcriptomics or single cell metabolomics [89], single cell proteomics can increase information on co- or anticorrelated proteins. This information can be used to accurately build protein sets and networks to adequately understand the impact of nutrition on human physiology [79].

In general, most of the scientific relationships that could be established between diet and human health and disease were achieved by epidemiological studies [90] but not by establishing a direct mechanistic link. The assignment of dietary patterns (for example high protein, low carbohydrates intake) to physiological outcome is complicated for various reasons. For example, co-occurrence of food ingredients and their potential interactions, the genetic and physiological background of the patient, and environmental factors are difficult to control. In practice, in human studies dietary intake of proteins and peptides is difficult to measure accurately [91]. Additionally, the amounts of food ingredients can vary due to seasonal or agronomic factors [92], which have often been ignored in epidemiological nutrition studies [93]. To support future studies, data on food composition including peptides are being established and stored in databases such as USDA National Nutrient Database (http://ndb.nal.usda.gov/).

\section{Gut, microbiome and nutrition}

So far we focused in this article on endogenously expressed proteins as potential biomarkers and on the potential roles of dietary proteins and peptides in the body. Here, we aim to enlarge our view on the effects of nutrition on physiology by considering three aspects: host organism, food, and in particular resident microorganisms, also known as the microbiome. Humans, like other higher organisms, are holobionts: multicellular hosts that live in interrelationship with bacteria, archaea, viruses, fungi, and other eukaryotes. Microbes in the gastro-intestinal tract colonize at the direct interface of the barrier of the human body formed by epithelial cells. The total number of genes of the various microbial species harbored by the intestine are estimated to exceed the number of our human genes by at least two orders of magnitude [94]. The intestinal microbiome consists of different "cell lineages" that communicate with each other and the host, and maintains and repairs itself by selfreplication. This microbiome armed with specific enzymes helps to digest food, consumes, stores and redistributes energy, and mediates physiologically important chemical transformations. Thus, the intestinal microbiome can be construed as a metabolically active "organ" affecting the host's metabolic capabilities, immunity, and pathogenesis $[95,96]$. For instance, enzymes of gut microbiota degrade a variety of dietary substances including plantderived pectin, cellulose, hemicellulose, and starches that are otherwise inaccessible to the host [97].

\subsection{Overview of an emerging nutriproteomic field}

The gut microbial community is established within the first 3 years of life. Human milk is known to protect newborns from infection due to several bioactive and immunological ingredients such as IgG [98], or upon digestion of lactoferrin released antimicrobial lactofericciin [99]. Also, such factors contribute to maturation of the infant intestine and companion microbial community. These examples indicate that gut microbiota and protein ingredients of nutrition affect human health.

Understanding principles and mechanisms of action of microbiota, including in particular the role of proteins of the microbiome, is key to improve human fitness by reshaping disease associated gastro-intestinal flora through intervention with, e.g. medical nutrition [100]. Furthermore protein or peptide biomarkers derived from the microbiome will probably be informative for specifically treating patients. Substantial first efforts undertaken by the Human Microbiome Project (HMP) and its precursor, the Human Gut Microbiome Initiative (HGMI), facilitated valuable insights into the composition of the adults gut microbiota. Due to technical feasibilities, these projects have focused mostly on phylogenetic analyses based on bacterial 16S rRNA sequencing [101]. The detailed composition of the microbiome is highly variable even between healthy individuals [102]. But notably most strains in the gut of an individual remain steadily residents for decades [103]. This striking fact argues for personalized nutritional or medical intervention based on individual microbiomes including metaproteomes, in addition to tailored nutrition strategies that are based on personal genetic information [104]. 
To cope with the unmanageable complexity of the three "omes", formed by nutrition, microbiota and the host, and to facilitate insights in their interplay, model systems were applied $[105,106]$. Germ free mice can be inoculated with a defined standard set of microorganisms or even a complete human microbiome, so called "humanized mice". For example, transplantation of intact human fecal microbiota from each member of an obesity discordant twin pair into separate groups of recipient germ-free mice has been successfully done. Feeding of these mice with defined chow resulted in a significantly greater increase in body mass and adiposity of mice that were inoculated with obese twin's microbiota compared to mice containing the lean co-twin's microbiota [107]. In another study germ-free mice were successfully inoculated with a defined set of microbes and their change in composition was monitored in response to defined nutritional components [108]. However, creating and housing such mice is not trivial and efforts are needed to control and standardize experiments with germ free mice [105].

In addition to metabolic effects, microbiomes seem to play a number of other roles. Perhaps most prominently, in the context of inflammatory bowel diseases mouse gut microbiota were found to be associated with inflammation at the intestinal barrier [109]. These unwanted effects can potentially be overcome by applying probiotics such as Lactobacillus casei. Notably, supplementation of amino acids such as glutamine in efficient formulations were proposed as a strategy to alleviate intestinal inflammation [110], which is currently being tested in clinical trials. Microorganisms in the gut will certainly play a role in the efficiency of this treatment approach.

In general, to improve the understanding of the role of microbiota in health and disease, a paradigm shift from traditional species identification and rather descriptive whole genome sequencing (WGS)-based metagenomics towards functional analysis will be required. In particular analyses of the entire proteome of microbiota, the metaproteome [111], will allow us to better understand the function of microbiomes in the human host and in processing of food.

\subsection{Metaproteomics methodologies}

Usually, metatranscriptomic and metaproteomic data analyses rely on comparisons to reference genomes $[112,113]$. However, the microbial diversity of the gastro-intestinal tract extends far beyond what is covered by reference databases, in part due to the difficulty of culturing many (anaerobic) microorganisms. Even though reference genomes for all microbial species would be available, the specific assignment of identified transcripts from next generation sequencing (NGS) and peptides from proteomic experiments remains difficult, especially for closely related species. Emerging single cell sequencing approaches [114] hold promise to achieve a sensitivity and data resolution of mixed communities on the single organism level, thus omitting the need of isolating and culturing each individual species separately.

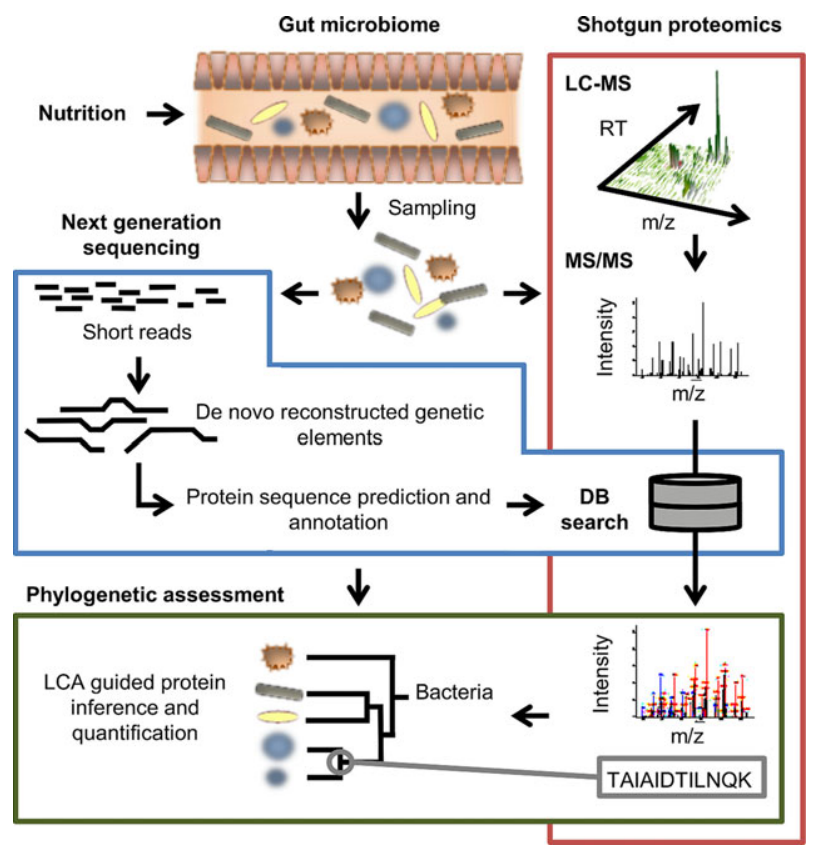

Figure 5. An emerging workflow for shotgun proteomic analysis of complex ecosystems such as the human or mouse intestine. In metaproteomics, proteins of multiple species are subjected to LC-MS/MS, whereas complementary next generation sequencing (NGS) data, such as RNA-sequencing data, are used to reconstruct protein sequences for peptide identification by a sequence database (DB) search engine. Thereby, the limitations due to sparse public sequence databases can be overcome. Phylogenetic binning of identifications needs to be performed in order to infer and quantify proteins properly with respect to the organism they may have derived from. One such tool for this purpose is the lowest common ancestor algorithm (LCA) [116].

However, methods including the lowest common ancestor algorithm (LCA), as implemented in MEGAN [115] and Unipept software [116], have been developed to determine the phylogenetic binning of transcript and peptide sequences retrieved from metagenomics, metatranscriptomics, and metaproteomics data, enabling to some extent a deeper resolution of meta-data by assigning identifications with respect to the organisms they may derive from. In addition the limitation due to sparse reference databases is likely to be overcome, as short read NGS data can be de novo assembled, which omits the need for mapping reads to reference genomes $[117,118]$. Such resources might become useful for the analysis of shotgun proteomic data, where sequence database searching is the most widespread technique to infer peptide sequences from mass spectral information [119]. Creating matched, sample specific databases for metaproteomics by using RNA-sequencing bears great potential for a more reliable and in depth analysis. These databases are more representative of the actual sample composition than incomplete genomic reference databases, and may thus perform better in terms of false identification (FDR) and protein inference [120]. Combining this "Proteomics Informed by 
Transcriptomics" (PIT) approach [121, 122] with aforementioned phylogenetic assessment is expected to greatly improve the informative value of metaproteomic studies (Fig. 5), whereby the entire expressed proteins of a microbiome can be in principle identified, characterized and quantified using high-precision mass spectrometry-based methods. Thereby, functionalities and activities of proteins derived from various microbial species may become identifiable for treating and preventing physiological deregulation by adaptation of nutrition.

\section{Human olfaction - essay on a paradigm for the complexities in nutrition research}

A number of physiological problems that are being tackled in nutrition research are difficult to analyze using reductionists' approaches - due to the inherent molecular complexity of diet and multifactorial molecular and physiological responses of the consumer. Targeting sensory-induced regulation of appetite is such a major, difficult to grasp subject of current nutrition research [123].

\subsection{Overview on odor perception}

Orthonasal odor perception of food influences appetite and seems to be a main factor of short-term over-eating. The nowadays increased availability of flavorful energy-rich foods is commonly attributed to the burst in prevalence of obesity. Notably, consumption of mother's milk can potentially support the establishment of preferences for food as a result of odorants derived from the acromastium of the mother $[124,125]$.

In general, retrogradal aroma stimulation can inhibit prolonged eating, resulting in earlier meal termination. Sensoryinduced manipulation can contribute to eating preferences and provide avenues to counteract phases of overeating [126]. However, after many years of intensive studies it is still largely unknown how the major (ca. up to 40) chemically diverse key food odorants from a group of ca. 230 out of ca. 10000 volatile compounds interact with our ca. 400 olfactory receptors [127]. About 35 odorant-receptor interactions were determined so far. Although odors can in principle be analyzed comprehensively and quantitatively in experimentally well-controlled setups, the number of possible combinations of odorants and proteins that serve as receptors is high. Moreover, mixing of various odorants for the creation of novel odor perceptions can lead to loss of individual contributions.

The rhodopsin-like $G$ protein coupled seven transmembrane helix receptors represent one of the largest gene/protein families in the human genome [128]. Structural information would allow for gaining insights in the molecular mechanisms of transducing chemical signals via the membrane of sensory neurons. But these proteins feature large hydrophilic domains that are very difficult to crystallize for structural (x-ray) analyses. So far success in this area has been low. Nevertheless, just recently the structure of opsin, a Gprotein coupled receptor of the retina, was solved to provide a template for modelling of olfactory receptor homology. This result now enables more efficient investigation of the structural basis of odorant-receptor interaction (Fig. 6A) [129].

Furthermore, functional model systems (such as standard mammalian cell lines that can be easily grown like HeLa), which express olfactor-type $G$ proteins can be used to analyze the mode of action of transducing a chemical signal. Conformational changes of receptor proteins can be detected in simplistic and combinatorial approaches (using various receptors and odorants/odorant mixtures). In general, once an odorant has bound to a receptor with more or less affinity, the receptor undergoes structural changes and activates the olfactory-type $\mathrm{G}$ protein on the inside of the sensory neuron. The olfactory G protein in turn activates a lyase-adenylate cyclase to convert ATP into cyclic AMP, which induces a cascade of internal signals, resulting in the development of action potential and electrical signaling of neurons (Fig. 6B). These signals are being converted by the Bulbus olfactorius and finally integrated by the limbic system and the hypothalamus (Fig. 6C).

Olfactory receptors have co-evolved with external chemical stimuli such as odorants derived from food. Evolutionary history might have played a role in determining and shaping our complex set-up of olfactory signaling networks and perception of odorants.

\subsection{Human olfaction as a paradigm for systems proteomics}

Single cell detection of chemical transduction including the analysis of calcium [130] and other cellular signaling events may help to produce quantitative and predictable models of signal transfer (Fig. 6C). Such models will potentially benefit from integrating single-receptor events, which can be translated in electrical patterns that eventually contribute to human perception. Single-cell arrays [131] with cells expressing olfactory-type $\mathrm{G}$ proteins may become suitable devices for producing an experimental set-up to follow up such demanding membrane protein research.

Furthermore, taste is essentially a complex vital phenomenon, which seems to be mainly determined by an interplay of olfactory receptors and a complex matrix of incoming, potentially very volatile odorants (Fig. 6C) [132]. One may argue that (subjective) taste can thus probably not be deduced from single factors, showing some of the limits of conventional reductionist approaches [133]. As physiological phenomena in general, taste may rather be a result of an emerging (network) property [134] of the ensemble of olfactory receptors and incoming molecules.

In principle, systematic network-establishing proteomic analyses will probably provide a more adequate model to 
A

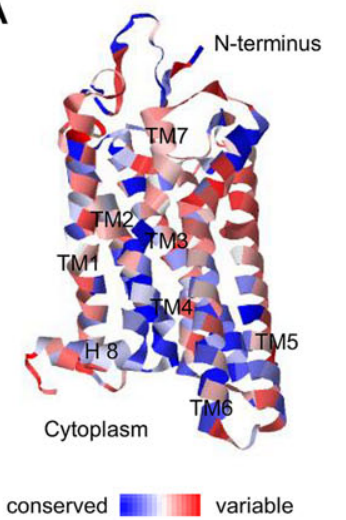

B
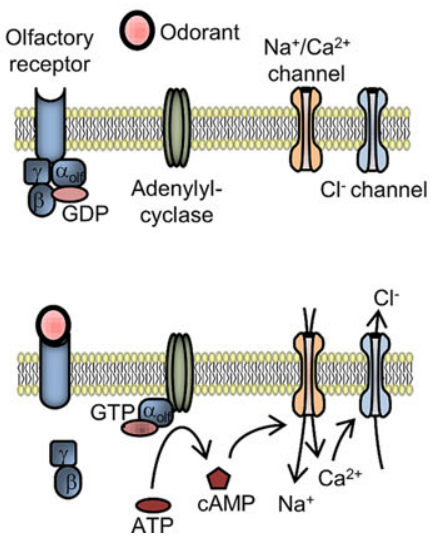

C

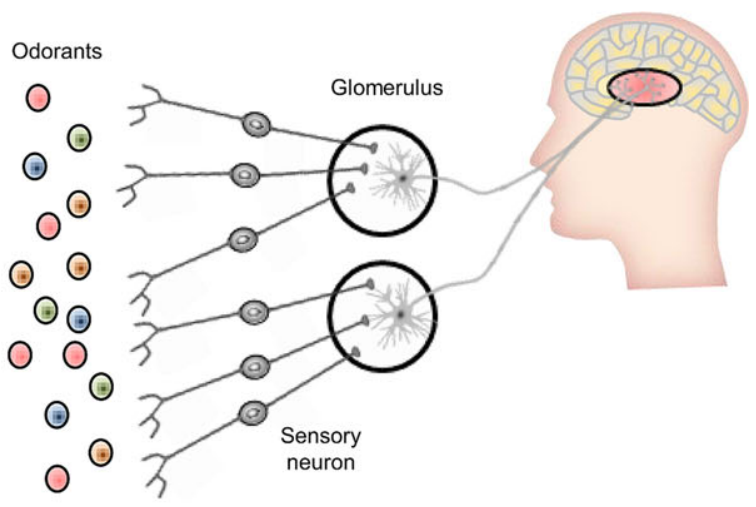

Figure 6. Human olfaction of food odorants as a paradigm for the inherent complexity of nutrition research. (A) The diverse odorants are recognized in sensory neurons via ca. 400 rhodopsin-like $\mathrm{G}$ protein coupled seven transmembrane helix receptors, the olfactory receptors. A multiple sequence alignment of 35 receptors, for which activating odorants were described [127], was constructed using TM-Coffee [156], and the degree of protein sequence conservation determined by calculating the Shannon Entropy [157]. Sequence variability was displayed on the opsin structure (PDB ID 4J40), a model for olfactory receptors [129], indicating structural regions that are probably important for ligand-receptor specificity. (B) Canonical knowledge was gained to understand transduction of chemical signals derived from defined molecules via olfactor-type $\mathrm{G}$ proteins to activate sensory neurons. (C) However, odorants from food come in as complex mixtures and signals are being transduced by various olfactor-type G proteins and sensory neurons. Complex neuronal circuits eventually produce neuronal perception to create emotion such as pleasant taste or disgust in the hypothalamus and the limbic system of the brain.

understand physiological processes such as modulation of taste. Analytical tools (including single cell proteomics methods [135]) as well as network biology concepts are becoming mature [136] to provide substantial insights in the establishment of taste preferences. Integration of data and cellular network analyses, analogously to currently extensively applied building of protein networks using amenable tools such as STRING [137] or Cytoscape [138], might help to gain a first view on complex phenomena such as taste. The development of suitable analytical frameworks may initially also benefit from using binary interaction data [139].

Furthermore, pattern (recognition) analyses are currently being applied to grasp complex traits such as cancer [78]. Pattern analyses may potentially contribute to a better understanding of transduction of chemical signals via protein receptors. Such knowledge might help to establish representation and perception of odorants - and food preferences.

In general, odorant input stimuli are projected onto a ductile neuronal net in the brain. Building connections between the neurons adapt to an odorant pattern in a learning process. This process induces self-organization of the neuronal network such that it better fits to the activation pattern. Underlying neural networks of biological processes such as olfaction and many other complex (e.g. emergent) phenomena can be analyzed by a number of software tools such as intuitive self-organizing maps (SOMs) $[140,141]$.

In general, knowledge about abundance and activity of olfactory receptors and their interaction with a multidimensional chemical environment will potentially allow us to grasp the complex physiology of food perception and taste preferences. This knowledge could be exploited for designing odor recombinants to target appetite regulation, for example to inhibit prolonged eating by retro-nasal aroma stimulation [142]. Eventually such knowledge will even hint to potential materialistic grounds of aesthetic phenomena such as taste.

The here sketched ideas seem very ambitious. Future technology developments in analytics and bioinformatics might eventually provide us with tools to dare answering fundamental questions from a materialistic point of view such as: how can food induce delight or distaste? The outcome from the here sketched research dealing with inherently complex and nonseparable aspects of biology remains speculative.

\section{Perspectives for nutriproteomics}

In principle, nutritional science can benefit a lot from sophisticated analyses of proteins. Clearly, technological advancements will remain important to overcome the abovediscussed current analytical limitations.

Integration of multiple layers of biological information such as genetic/epigenetic variation, gene expression and metabolites in pathways and regulatory networks may ultimately result in adequate and reproducible outputs for detecting physiological changes. In particular during prenatal stages and early postnatal life, i.e. during the period of cell differentiation and specific tissue formation, plasticity of physiology is highly sensitive to environmental factors such as nutrients and stressors [143], arguing for intensifying research in this direction to address the problem by the root. Interestingly, physiological effects of diets with highand low-protein content, as well as protein sources (animals or plants), seem to be age-dependent [144]. An in-depth 
proteome wide investigation would potentially shed additional light to underlying processes, to explain and streamline nutrition to the needs of different (physiological) ages. Another emerging topic of nutritional science consists of the relationship between circadian biology, food intake and metabolism, in which posttranslational protein signalling seems to play a major, so far largely unexplored role [145].

To increase relevance of nutriproteomics research, future studies will certainly have to focus even more towards application in humans. Such studies will be important to not only understand the mode of action of diet by correlation or association analyses but to get deeper insights in the underlying causalities. These causalities do not necessarily have to be reduced to single pieces. Instead causality structures can be established from complex data by using sophisticated algorithms, as shown recently, for example by Sugihara, May and co-workers [146].

Lessons learned from large-scale human nutritional intervention studies that made extensive usage of genomics-based analyses such as DIOGENES (Diet, Obesity, and Genes) [147] or metabolomics studies such as of Stanstrup et al. [148] may provide a good basis for identifying functional links in protein-based data. Suitable study design (in the best case double-blind placebo-controlled crossover), using wellstratified human cohorts including consideration of gender [149], age [144] and other issues, and standardization of diets is crucial to gain sufficient power to detect subtle effects during nutritional interventions or in longitudinal studies. This requirement is not at all trivial given the complexities to monitor human studies over a longer time range.

Finally, we may argue that digging too deeply in molecular details of biological (vital) phenomena may in some contexts potentially rather result in a loss instead of a gain of useful information. Focusing more intensively on designing mathematical structures of the phenomenon under study may provide a testable fruitful approach to tackle complex problems - in nutrition research and in physiology.

Our work was supported by the German Ministry for Education and Research (BMBF, grant number 0315082, 01EA1303 to S.S.), the European Union (FP7/2007-2013), under grant agreement $n^{\circ} 262055$ (ESGI)), and the Max Planck Society. This work is part of the Ph.D. thesis of T.L.

The authors have declared no conflict of interest.

\section{References}

[1] World Health Organization (WHO), Fact sheet $\mathrm{N}^{\circ}$ 311: obesity and overweight. 2014. http://www.who.int/ mediacentre/factsheets/fs311/en/.

[2] Muller, M., Kersten, S., Nutrigenomics: goals and strategies. Nat. Rev. Genet. 2003, 4, 315-322.

[3] Rist, M. J., Wenzel, U., Daniel, H., Nutrition and food science go genomic. Trends Biotechnol. 2006, 24, 172-178.
[4] Potischman, N., Weed, D. L., Causal criteria in nutritional epidemiology. Am. J. Clin. Nutr. 1999, 69, 1309S-1314S.

[5] Ley, S. H., Hamdy, O., Mohan, V., Hu, F. B., Prevention and management of type 2 diabetes: dietary components and nutritional strategies. Lancet 2014, 383, 1999-2007.

[6] Kussmann, M., Panchaud, A., Affolter, M., Proteomics in nutrition: status quo and outlook for biomarkers and bioactives. J. Proteome Res. 2010, 9, 4876-4887.

[7] Domon, B., Aebersold, R., Mass spectrometry and protein analysis. Science 2006, 312, 212-217.

[8] Afman, L., Muller, M., Nutrigenomics: from molecular nutrition to prevention of disease. J. Am. Diet. Assoc. 2006, 106, 569-576.

[9] Pawankar, R., Canonica, G. W., Holgate, S. T., Lockey, R. F., WAO White Book on Allergy, World Allergy Organization, Milwaukee, WI, USA 2011.

[10] Cianferoni, A., Spergel, J. M., Food allergy: review, classification and diagnosis. Allergol. Int. 2009, 58, 457-466.

[11] Koeberl, M., Clarke, D., Lopata, A. L., Next generation of food allergen quantification using mass spectrometric systems. J. Proteome Res. 2014, 13, 3499-3509.

[12] Heick, J., Fischer, M., Kerbach, S., Tamm, U., Popping, B., Application of a liquid chromatography tandem mass spectrometry method for the simultaneous detection of seven allergenic foods in flour and bread and comparison of the method with commercially available ELISA test kits. J. AOAC Int. 2011, 94, 1060-1068.

[13] Liebler, D. C., Zimmerman, L. J., Targeted quantitation of proteins by mass spectrometry. Biochemistry 2013, 52, 3797-3806.

[14] Nakamura, R., Teshima, R., Immunoproteomics analysis of food allergens. Methods Mol. Biol. 2014, 1072, 725-735.

[15] Gasilova, N., Girault, H. H., Component-resolved diagnostic of cow's milk allergy by immunoaffinity capillary electrophoresis-matrix assisted laser desorption/ionization mass spectrometry. Anal. Chem. 2014, 86, 63376345.

[16] Fiedler, K. L., McGrath, S. C., Callahan, J. H., Ross, M. M., Characterization of grain-specific Peptide markers for the detection of gluten by mass spectrometry. J. Agric. Food Chem. 2014, 62, 5835-5844.

[17] Dell, A., Morris, H. R., Glycoprotein structure determination by mass spectrometry. Science 2001, 291, 2351-2356.

[18] Chandra, R. K., Food hypersensitivity and allergic disease: a selective review. Am. J. Clin. Nutr. 1997, 66, 526S-529S.

[19] Dam, S., Thaysen-Andersen, M., Stenkjaer, E., Lorentzen, A. et al., Combined $\mathrm{N}$-glycome and $\mathrm{N}$-glycoproteome analysis of the lotus japonicus seed globulin fraction shows conservation of protein structure and glycosylation in legumes. $J$. Proteome Res. 2013, 12, 3383-3392.

[20] Kitts, D. D., Weiler, K., Bioactive proteins and peptides from food sources. Applications of bioprocesses used in isolation and recovery. Curr. Pharm. Des. 2003, 9, 1309-1323.

[21] Moller, N. P., Scholz-Ahrens, K. E., Roos, N., Schrezenmeir, J., Bioactive peptides and proteins from foods: indication for health effects. Eur. J. Nutr. 2008, 47, 171-182. 
[22] Minkiewicz, P., Dziuba, J., Iwaniak, A., Dziuba, M., Darewicz, M., BIOPEP database and other programs for processing bioactive peptide sequences. J. AOAC Int. 2008, 91, 965980.

[23] Severin, S., Wenshui, X., Milk biologically active components as nutraceuticals: review. Crit. Rev. Food Sci. Nutr. 2005, 45, 645-656.

[24] Madureira, A. R., Pereira, C. I., Gomes, A. M. P., Pintado, M. E., Malcata, F. X., Bovine whey proteins - overview on their main biological properties. Food Res. Int. 2007, 40, 1197-1211.

[25] D'Auria, E., Agostoni, C., Giovannini, M., Riva, E. et al., Proteomic evaluation of milk from different mammalian species as a substitute for breast milk. Acta. Paediatr. 2005, 94, 1708-1713.

[26] Seppo, L., Jauhiainen, T., Poussa, T., Korpela, R., A fermented milk high in bioactive peptides has a blood pressure-lowering effect in hypertensive subjects. Am. J. Clin. Nutr. 2003, 77, 326-330.

[27] Smacchi, E., Gobbetti, M., Bioactive peptides in dairy products: synthesis and interaction with proteolytic enzymes. Food Microbiol. 2000, 17, 129-141.

[28] Matar, C., Amiot, J., Savoie, L., Goulet, J., The effect of milk fermentation by Lactobacillus helveticus on the release of peptides during in vitro digestion. J. Dairy Sci. 1996, 79, 971-979.

[29] Argyri, K., Miller, D. D., Glahn, R. P., Zhu, L., Kapsokefalou, M., Peptides isolated from in vitro digests of milk enhance iron uptake by caco-2 cells. J. Agric. Food Chem. 2007, 55, 10221-10225.

[30] Farnaud, S., Evans, R. W., Lactoferrin-a multifunctional protein with antimicrobial properties. Mol. Immunol. 2003, 40, 395-405.

[31] Panchaud, A., Kussmann, M., Affolter, M., Rapid enrichment of bioactive milk proteins and iterative, consolidated protein identification by multidimensional protein identification technology. Proteomics 2005, 5, 3836-3846.

[32] Meisel, H., Multifunctional peptides encrypted in milk proteins. Biofactors 2004, 21, 55-61.

[33] Baum, F., Fedorova, M., Ebner, J., Hoffmann, R., Pischetsrieder, M., Analysis of the endogenous peptide profile of milk: identification of 248 mainly casein-derived peptides. J. Proteome Res. 2013, 12, 5447-5462.

[34] Clare, D. A., Swaisgood, H. E., Bioactive milk peptides: a prospectus. J. Dairy Sci. 2000, 83, 1187-1195.

[35] Bellamy, W., Takase, M., Wakabayashi, H., Kawase, K., Tomita, M., Antibacterial spectrum of lactoferricin B, a potent bactericidal peptide derived from the $\mathrm{N}$-terminal region of bovine lactoferrin. J. Appl. Bacteriol. 1992, 73, 472479.

[36] Benkerroum, N., Antimicrobial peptides generated from milk proteins: a survey and prospects for application in the food industry. A review. Int. J. Dairy Technol. 2010, 63, 320-338.

[37] FitzGerald, R. J., Murray, B. A., Walsh, D. J., Hypotensive peptides from milk proteins. J. Nutr. 2004, 134, 980S988S.
[38] Jäkälä, P., Vapaatalo, H., Antihypertensive peptides from milk proteins. Pharmaceuticals 2010, 3, 251-272.

[39] Politis, I., Chronopoulou, R., Milk peptides and immune response in the neonate. Adv. Exp. Med. Biol. 2008, 606, 253269.

[40] Huang, S.-M., Chen, K.-N., Chen, Y.-P., Hong, W.-S., Chen, M.-J., Immunomodulatory properties of the milk whey products obtained by enzymatic and microbial hydrolysis. Int. J. Food Sci. Tech. 2010, 45, 1061-1067.

[41] Trivedi, M. S., Shah, J. S., Al-Mughairy, S., Hodgson, N. W. et al., Food-derived opioid peptides inhibit cysteine uptake with redox and epigenetic consequences. J. Nutr. Biochem. 2014, 25, 1011-1018.

[42] Ferranti, P., Traisci, M. V., Picariello, G., Nasi, A. et al., Casein proteolysis in human milk: tracing the pattern of casein breakdown and the formation of potential bioactive peptides. J. Dairy Res. 2004, 71, 74-87.

[43] Christensen, B., Schack, L., Klaning, E., Sorensen, E. S., Osteopontin is cleaved at multiple sites close to its integrinbinding motifs in milk and is a novel substrate for plasmin and cathepsin D. J. Biol. Chem. 2010, 285, 7929-7937.

[44] Guerrero, A., Dallas, D. C., Contreras, S., Chee, S. et al., Mechanistic peptidomics: factors that dictate the specificity on the formation of endogenous peptides in human milk. Mol. Cell. Proteomics 2014, DOI 10.1074/mcp.M113.036194.

[45] Dallas, D. C., Guerrero, A., Khaldi, N., Castillo, P. A. et al., Extensive in vivo human milk peptidomics reveals specific proteolysis yielding protective antimicrobial peptides. J. Proteome Res. 2013, 12, 2295-2304.

[46] Peng, K., Vucetic, S., Radivojac, P., Brown, C. J. et al., Optimizing long intrinsic disorder predictors with protein evolutionary information. J. Bioinform. Comput. Biol. 2005, 3, 35-60.

[47] Gao, L., Wang, A., Li, X., Dong, K. et al., Wheat quality related differential expressions of albumins and globulins revealed by two-dimensional difference gel electrophoresis (2-D DIGE). J. Proteomics 2009, 73, 279-296.

[48] Erickson, B. E., Proteomics data back up soy health claims. J Proteome Res 2005, 4, 219.

[49] Krishnan, H. B., Oehrle, N. W., Natarajan, S. S., A rapid and simple procedure for the depletion of abundant storage proteins from legume seeds to advance proteome analysis: a case study using Glycine max. Proteomics 2009, 9, 31743188.

[50] Park, J. H., Jeong, H. J., de Lumen, B. O., Contents and bioactivities of lunasin, bowman-birk inhibitor, and isoflavones in soybean seed. J. Agric. Food Chem. 2005, $53,7686-7690$

[51] de Lumen, B. O., Lunasin: a novel cancer preventive seed peptide that modifies chromatin. J. AOAC Int. 2008, 91, 932935.

[52] Sakata, K., Ohyanagi, H., Nobori, H., Nakamura, T. et al., Soybean proteome database: a data resource for plant differential omics. J. Proteome Res. 2009, 8, 3539-3548.

[53] Munro, I. C., Harwood, M., Hlywka, J. J., Stephen, A. M. et al., Soy isoflavones: a safety review. Nutr. Rev. 2003, 61, $1-33$. 
[54] Gestetner, B., Birk, Y., Tencer, Y., oybean saponins. Fate of ingested soybean saponins and the physiological aspect of their hemolytic activity. J. Agric. Food Chem. 1968, 16, 1031-1035.

[55] Beermann, C., Euler, M., Herzberg, J., Stahl, B., Antioxidative capacity of enzymatically released peptides from soybean protein isolate. Eur. Food Res. Technol. 2009, 229, 637-644.

[56] Danchenko, M., Skultety, L., Rashydov, N. M., Berezhna, V. V. et al., Proteomic analysis of mature soybean seeds from the Chernobyl area suggests plant adaptation to the contaminated environment. J. Proteome Res. 2009, 8, 29152922.

[57] Bourgeois, M., Jacquin, F., Savois, V., Sommerer, N. et al., Dissecting the proteome of pea mature seeds reveals the phenotypic plasticity of seed protein composition. Proteomics 2009, 9, 254-271.

[58] Kussmann, M., Affolter, M., Nagy, K., Holst, B., Fay, L. B., Mass spectrometry in nutrition: understanding dietary health effects at the molecular level. Mass Spectrom. Rev. 2007, 26, 727-750.

[59] Frank, A., Pevzner, P., PepNovo: de novo peptide sequencing via probabilistic network modeling. Anal. Chem. 2005, 77, 964-973.

[60] Zhang, J., Xin, L., Shan, B., Chen, W. et al., PEAKS DB: de novo sequencing assisted database search for sensitive and accurate peptide identification. Mol. Cell. Proteomics 2012, 11, M111.010587.

[61] Cox, J., Neuhauser, N., Michalski, A., Scheltema, R. A. et al., Andromeda: a peptide search engine integrated into the MaxQuant environment. J. Proteome Res. 2011, 10, 17941805.

[62] Munch, J., Standker, L., Forssmann, W. G., Kirchhoff, F., Discovery of modulators of HIV-1 infection from the human peptidome. Nat. Rev. Microbiol. 2014, 12, 715722.

[63] Kim, Y. J., Gallien, S., van Oostrum, J., Domon, B., Targeted proteomics strategy applied to biomarker evaluation. Proteomics Clin. Appl. 2013, 7, 739-747.

[64] Omenn, G. S., On best practices: the Institute of Medicine scheme for developing, validating, and demonstrating clinical utility of omics-based diagnostic and predictive tests. Proteomics Clin. Appl. 2013, 7, 748-755.

[65] Kussmann, M., Affolter, M., Fay, L. B., Proteomics in nutrition and health. Comb. Chem. High Throughput Screen 2005, 8, 679-696.

[66] Adam, B. L., Vlahou, A., Semmes, O. J., Wright, G. L., Jr., Proteomic approaches to biomarker discovery in prostate and bladder cancers. Proteomics 2001, 1, 12641270.

[67] Parker, C. E., Pearson, T. W., Anderson, N. L., Borchers, C. H., Mass-spectrometry-based clinical proteomics-a review and prospective. Analyst 2010, 135, 1830-1838.

[68] Roberts, L. D., Koulman, A., Griffin, J. L., Towards metabolic biomarkers of insulin resistance and type 2 diabetes: progress from the metabolome. Lancet Diabetes Endocrinol. 2014, 2, 65-75.
[69] van Ommen, B., Keijer, J., Heil, S. G., Kaput, J., Challenging homeostasis to define biomarkers for nutrition related health. Mol. Nutr. Food Res. 2009, 53, 795-804.

[70] Senechal, S., Kussmann, M., Nutriproteomics: technologies and applications for identification and quantification of biomarkers and ingredients. Proc. Nutr. Soc. 2011, 70, 351-364.

[71] Breikers, G., van Breda, S. G., Bouwman, F. G., van Herwijnen, M. H. et al., Potential protein markers for nutritional health effects on colorectal cancer in the mouse as revealed by proteomics analysis. Proteomics 2006, 6, 2844-2852.

[72] Herzog, A., Kindermann, B., Doring, F., Daniel, H., Wenzel, U., Pleiotropic molecular effects of the pro-apoptotic dietary constituent flavone in human colon cancer cells identified by protein and mRNA expression profiling. Proteomics 2004, 4, 2455-2464.

[73] Knowles, L. M., Milner, J. A., Diallyl disulfide induces ERK phosphorylation and alters gene expression profiles in human colon tumor cells. J. Nutr. 2003, 133, 2901-2906.

[74] Dinkova-Kostova, A. T., Holtzclaw, W. D., Cole, R. N., Itoh, K. et al., Direct evidence that sulfhydryl groups of Keap1 are the sensors regulating induction of phase 2 enzymes that protect against carcinogens and oxidants. Proc. Natl. Acad. Sci. U.S.A. 2002, 99, 11908-11913.

[75] Renes, J., Rosenow, A., Roumans, N., Noben, J. P., Mariman, E. C., Calorie restriction-induced changes in the secretome of human adipocytes, comparison with resveratrolinduced secretome effects. Biochim. Biophys. Acta. 2014, 1844, 1511-1522.

[76] Freiwald, A., Weidner, C., Witzke, A., Huang, S. Y. et al., Comprehensive proteomic data sets for studying adipocyte-macrophage cell-cell communication. Proteomics 2013, 13, 3424-3428.

[77] Meierhofer, D., Weidner, C., Hartmann, L., Mayr, J. A. et al., Protein sets define disease States and predict in vivo effects of drug treatment. Mol. Cell. Proteomics 2013, 12, 19651979.

[78] Vogelstein, B., Papadopoulos, N., Velculescu, V. E., Zhou, S. et al., Cancer genome landscapes. Science 2013, 339, $1546-1558$.

[79] Sauer, S., Protein set analyses: how could this impact the clinic? Expert Rev. Proteomics 2013, 10, 305-307.

[80] Meierhofer, D., Weidner, C., Sauer, S., Integrative analysis of transcriptomics, proteomics, and metabolomics data of white adipose and liver tissue of high-fat diet and rosiglitazone-treated insulin-resistant mice identified pathway alterations and molecular hubs. J. Proteome Res. 2014, $13,5592-5602$.

[81] Chen, T., Dent, S. Y., Chromatin modifiers and remodellers: regulators of cellular differentiation. Nat. Rev. Genet. 2014, 15, 93-106.

[82] Ganesh, V., Hettiarachchy, N. S., Nutriproteomics: a promising tool to link diet and diseases in nutritional research. Biochim. Biophys. Acta. 2012, 1824, 1107-1117.

[83] Sauer, S., Lange, B. M., Gobom, J., Nyarsik, L. et al., Miniaturization in functional genomics and proteomics. Nat. Rev. Genet. 2005, 6, 465-476. 
[84] Anderson, N. L., Anderson, N. G., The human plasma proteome: history, character, and diagnostic prospects. Mol. Cell. Proteomics 2002, 1, 845-867.

[85] Altelaar, A. F., Munoz, J., Heck, A. J., Next-generation proteomics: towards an integrative view of proteome dynamics. Nat. Rev. Genet. 2013, 14, 35-48.

[86] Olsen, J. V., Mann, M., Status of large-scale analysis of posttranslational modifications by mass spectrometry. Mol. Cell. Proteomics 2013, 12, 3444-3452.

[87] Weidner, C., Fischer, C., Sauer, S., PHOXTRACK - a tool for interpreting comprehensive data sets of posttranslational modifications of proteins. Bioinformatics 2014, 30, 34103411.

[88] Shapiro, E., Biezuner, T., Linnarsson, S., Single-cell sequencing-based technologies will revolutionize wholeorganism science. Nat. Rev. Genet. 2013, 14, 618-630.

[89] Zenobi, R., Single-cell metabolomics: analytical and biological perspectives. Science 2013, 342, 1243259.

[90] Boeing, H., Nutritional epidemiology: new perspectives for understanding the diet-disease relationship? Eur. J. Clin. Nutr. 2013, 67, 424-429.

[91] van Nielen, M., Feskens, E. J., Mensink, M., Sluijs, I. et al., Dietary protein intake and incidence of type 2 diabetes in Europe: the EPIC-InterAct Case-Cohort Study. Diabetes Care 2014, 37, 1854-1862.

[92] Buxton, D. R., Quality-related characteristics of forages as influenced by plant environment and agronomic factors. Anim. Feed Sci. Technol. 1996, 59, 37-49.

[93] Riboli, E., Slimani, N., Kaaks, R., Identifiability of food components for cancer chemoprevention. IARC Sci. Publ. 1996, 23-31.

[94] Backhed, F., Ley, R. E., Sonnenburg, J. L., Peterson, D. A., Gordon, J. I., Host-bacterial mutualism in the human intestine. Science 2005, 307, 1915-1920.

[95] Macpherson, A. J., Harris, N. L., Interactions between commensal intestinal bacteria and the immune system. Nat. Rev. Immunol. 2004, 4, 478-485.

[96] Turnbaugh, P. J., Ley, R. E., Mahowald, M. A., Magrini, V. et al., An obesity-associated gut microbiome with increased capacity for energy harvest. Nature 2006, 444, 10271031.

[97] Savage, D. C., Gastrointestinal microflora in mammalian nutrition. Annu. Rev. Nutr. 1986, 6, 155-178.

[98] Ruiz-Palacios, G. M., Calva, J. J., Pickering, L. K., LopezVidal, Y. et al., Protection of breast-fed infants against Campylobacter diarrhea by antibodies in human milk. J. Pediatr. 1990, 116, 707-713.

[99] Jones, E. M., Smart, A., Bloomberg, G., Burgess, L., Millar, M. R., Lactoferricin, a new antimicrobial peptide. J. Appl. Bacteriol. 1994, 77, 208-214.

[100] Kussmann, M., Fay, L. B., Nutrigenomics and personalized nutrition: science and concept. Pers. Med. 2008, 5, 447455.

[101] Blaut, M., Ecology and physiology of the intestinal tract. Curr. Top Microbiol. Immunol. 2013, 358, 247-272.
[102] Human Microbiome Project, C., Structure, function and diversity of the healthy human microbiome. Nature 2012, 486, 207-214.

[103] Faith, J. J., Guruge, J. L., Charbonneau, M., Subramanian, $\mathrm{S}$. et al., The long-term stability of the human gut microbiota. Science 2013, 341, 1237439.

[104] Mutch, D. M., Wahli, W., Williamson, G., Nutrigenomics and nutrigenetics: the emerging faces of nutrition. Faseb. J. 2005, 19, 1602-1616.

[105] Cassidy, L., Tholey, A., Model organism proteomics as a tool for the study of host-microbiome interactions. Proteomics Clin. Appl. 2014, 8, 665-676.

[106] Tholey, A., Treitz, C., Kussmann, M., Bendixen, E. et al., Model organisms proteomics-from holobionts to human nutrition. Proteomics 2013, 13, 2537-2541.

[107] Ridaura, V. K., Faith, J. J., Rey, F. E., Cheng, J. et al., Gut microbiota from twins discordant for obesity modulate metabolism in mice. Science 2013, 341, 1241214.

[108] Faith, J. J., McNulty, N. P., Rey, F. E., Gordon, J. I., Predicting a human gut microbiota's response to diet in gnotobiotic mice. Science 2011, 333, 101-104.

[109] Nell, S., Suerbaum, S., Josenhans, C., The impact of the microbiota on the pathogenesis of IBD: lessons from mouse infection models. Nat. Rev. Microbiol. 2010, 8, 564-577.

[110] Coeffier, M., Marion-Letellier, R., Dechelotte, P., Potential for amino acids supplementation during inflammatory bowel diseases. Inflamm. Bowel. Dis. 2010, 16, 518-524.

[111] Kolmeder, C. A., de Vos, W. M., Metaproteomics of our microbiome - developing insight in function and activity in man and model systems. J. Proteomics 2014, 97, 3-16.

[112] Maurice, C. F., Haiser, H. J., Turnbaugh, P. J., Xenobiotics shape the physiology and gene expression of the active human gut microbiome. Cell 2013, 152, 39-50.

[113] Haange, S. B., Oberbach, A., Schlichting, N., Hugenholtz, F. et al., Metaproteome analysis and molecular genetics of rat intestinal microbiota reveals section and localization resolved species distribution and enzymatic functionalities. J. Proteome Res. 2012, 11, 5406-5417.

[114] Metzker, M. L., Sequencing technologies - the next generation. Nat. Rev. Genet. 2010, 11, 31-46.

[115] Huson, D. H., Auch, A. F., Qi, J., Schuster, S. C., MEGAN analysis of metagenomic data. Genome Res. 2007, 17, 377386.

[116] Mesuere, B., Devreese, B., Debyser, G., Aerts, M. et al., Unipept: tryptic peptide-based biodiversity analysis of metaproteome samples. J. Proteome Res. 2012, 11, 57735780

[117] Haas, B. J., Papanicolaou, A., Yassour, M., Grabherr, M. et al., De novo transcript sequence reconstruction from RNA-seq using the Trinity platform for reference generation and analysis. Nat. Protoc. 2013, 8, 1494-1512.

[118] Schulz, M. H., Zerbino, D. R., Vingron, M., Birney, E., Oases: robust de novo RNA-seq assembly across the dynamic range of expression levels. Bioinformatics 2012, 28, 10861092. 
[119] Nesvizhskii, A. I., A survey of computational methods and error rate estimation procedures for peptide and protein identification in shotgun proteomics. J. Proteomics 2010, 73, 2092-2123.

[120] Tanca, A., Palomba, A., Deligios, M., Cubeddu, T. et al., Evaluating the impact of different sequence databases on metaproteome analysis: insights from a lab-assembled microbial mixture. PLoS One 2013, 8, e82981.

[121] Evans, V. C., Barker, G., Heesom, K. J., Fan, J. et al., De novo derivation of proteomes from transcriptomes for transcript and protein identification. Nat. Methods 2012, 9, 12071211.

[122] Luge, T., Kube, M., Freiwald, A., Meierhofer, D. et al., Transcriptomics assisted proteomic analysis of Nicotiana occidentalis infected by 'Candidatus Phytoplasma mali' strain AT. Proteomics 2014, 14, 1882-1889.

[123] Rangel, A., Regulation of dietary choice by the decisionmaking circuitry. Nat. Neurosci. 2013, 16, 1717-1724.

[124] Kupferschmidt, K., FOOD SCIENCE following the flavor. Science $2013,340,808-809$.

[125] Lausten-Thomsen, U., Bille, D. S., Nasslund, I., Folskov, L. et al., Neonatal anthropometrics and correlation to childhood obesity-data from the Danish Children's Obesity Clinic. Eur. J. Pediatr. 2013, 172, 747-751.

[126] Stroebele, N., De Castro, J. M., Effect of ambience on food intake and food choice. Nutrition 2004, 20, 821-838.

[127] Dunkel, A., Steinhaus, M., Kotthoff, M., Nowak, B. et al., Nature's chemical signatures in human olfaction: a foodborne perspective for future biotechnology. Angew. Chem. Int. Ed. Engl. 2014, 53, 7124-7143.

[128] Krautwurst, D., Human olfactory receptor families and their odorants. Chem. Biodivers. 2008, 5, 842-852.

[129] Park, J. H., Morizumi, T., Li, Y., Hong, J. E. et al., Opsin, a structural model for olfactory receptors? Angew. Chem. Int. Ed. Engl. 2013, 52, 11021-11024.

[130] Stosiek, C., Garaschuk, O., Holthoff, K., Konnerth, A., In vivo two-photon calcium imaging of neuronal networks. Proc. Natl. Acad. Sci. U.S.A. 2003, 100, 7319-7324.

[131] Dykstra, B., Ramunas, J., Kent, D., McCaffrey, L. et al., High-resolution video monitoring of hematopoietic stem cells cultured in single-cell arrays identifies new features of self-renewal. Proc. Natl. Acad. Sci. U.S.A. 2006, 103, 81858190.

[132] Krautwurst, D., Kotthoff, M., A hit map-based statistical method to predict best ligands for orphan olfactory receptors: natural key odorants versus "lock picks". Methods Mol. Biol. 2013, 1003, 85-97.

[133] Sauer, S., Diagnostic proteomics: will this impact the clinic? Proteomics Clin. Appl. 2013, 7, 725-726.

[134] Goh, K. I., Cusick, M. E., Valle, D., Childs, B. et al., The human disease network. Proc. Natl. Acad. Sci. U.S.A. 2007, 104, 8685-8690.

[135] Salehi-Reyhani, A., Kaplinsky, J., Burgin, E., Novakova, M. et al., A first step towards practical single cell proteomics: a microfluidic antibody capture chip with TIRF detection. Lab Chip 2011, 11, 1256-1261.
[136] Vidal, M., Cusick, M. E., Barabasi, A. L., Interactome networks and human disease. Cell 2011, 144, 986-998.

[137] Franceschini, A., Szklarczyk, D., Frankild, S., Kuhn, M. et al., STRING v9.1: protein-protein interaction networks, with increased coverage and integration. Nucleic Acids Res. 2013, 41, D808-D815.

[138] Shannon, P., Markiel, A., Ozier, O., Baliga, N. S. et al., Cytoscape: a software environment for integrated models of biomolecular interaction networks. Genome Res. 2003, 13, 2498-2504.

[139] Braun, P., Interactome mapping for analysis of complex phenotypes: insights from benchmarking binary interaction assays. Proteomics 2012, 12, 1499-1518.

[140] Wirth, H., Loffler, M., von Bergen, M., Binder, H., Expression cartography of human tissues using self organizing maps. BMC Bioinformatics 2011, 12, 306.

[141] Tamayo, P., Slonim, D., Mesirov, J., Zhu, Q. et al., Interpreting patterns of gene expression with self-organizing maps: methods and application to hematopoietic differentiation. Proc. Natl. Acad. Sci. U.S.A. 1999, 96, 2907-2912.

[142] Snoek, H. M., Huntjens, L., van Gemert, L. J., De Graaf, C., Weenen, H., Sensory-specific satiety in obese and normalweight women. Am. J. Clin. Nutr. 2004, 80, 823-831.

[143] Barouki, R., Gluckman, P. D., Grandjean, P., Hanson, M., Heindel, J. J., Developmental origins of non-communicable disease: implications for research and public health. Environ. Health 2012, 11, 42.

[144] Levine, M. E., Suarez, J. A., Brandhorst, S., Balasubramanian, P. et al., Low protein intake is associated with a major reduction in IGF-1, cancer, and overall mortality in the 65 and younger but not older population. Cell. Metab. 2014, 19, 407-417.

[145] Mauvoisin, D., Dayon, L., Gachon, F., Kussmann, M., Proteomics and circadian rhythms: it's all about signaling! Proteomics 2014, DOI: 10.1002/pmic.201400187.

[146] Sugihara, G., May, R., Ye, H., Hsieh, C. H. et al., Detecting causality in complex ecosystems. Science 2012, 338, 496500.

[147] Larsen, T. M., Dalskov, S. M., van Baak, M., Jebb, S. A. et al., Diets with high or low protein content and glycemic index for weight-loss maintenance. New Engl. J. Med. 2010, 363, 2102-2113.

[148] Stanstrup, J., Schou, S. S., Holmer-Jensen, J., Hermansen, K., Dragsted, L. O., Whey protein delays gastric emptying and suppresses plasma fatty acids and their metabolites compared to casein, gluten, and fish protein. J. Proteome Res. 2014, 13, 2396-2408.

[149] Xie, G., Ma, X., Zhao, A., Wang, C. et al., The metabolite profiles of the obese population are gender-dependent. J. Proteome Res. 2014, 13, 4062-4073.

[150] Freiwald, A., Mao, L., Kodelja, V., Kliem, M. et al., Differential analysis of Crohn's disease and ulcerative colitis by mass spectrometry. Inflamm. Bowel Dis. 2011, 17, 1051-1052.

[151] Baumann, S., Ceglarek, U., Fiedler, G. M., Lembcke, J. et al., Standardized approach to proteome profiling of human serum based on magnetic bead separation and 
matrix-assisted laser desorption/ionization time-of-flight mass spectrometry. Clin. Chem. 2005, 51, 973-980.

[152] Jacobs, J. M., Adkins, J. N., Qian, W. J., Liu, T. et al., Utilizing human blood plasma for proteomic biomarker discovery. J. Proteome Res. 2005, 4, 1073-1085.

[153] Wang, T. J., Gona, P., Larson, M. G., Tofler, G. H. et al., Multiple biomarkers for the prediction of first major cardiovascular events and death. N. Engl. J. Med. 2006, 355, 2631-2639.

[154] Stastna, M., Van Eyk, J. E., Secreted proteins as a fundamental source for biomarker discovery. Proteomics 2012, $12,722-735$
[155] Mischak, H., loannidis, J. P., Argiles, A., Attwood, T. K. et al., Implementation of proteomic biomarkers: making it work. Eur. J. Clin. Invest. 2012, 42, 1027-1036.

[156] Chang, J. M., Di Tommaso, P., Taly, J. F., Notredame, C., Accurate multiple sequence alignment of transmembrane proteins with PSI-Coffee. BMC Bioinformatics 2012, 13(Suppl 4), S1.

[157] Garcia-Boronat, M., Diez-Rivero, C. M., Reinherz, E. L., Reche, P. A., PVS: a web server for protein sequence variability analysis tuned to facilitate conserved epitope discovery. Nucleic Acids Res. 2008, 36, W3541. 